\title{
Analytic Renormalization Using Many Space-Time Dimensions
}

\author{
Eugene R. Speer * \\ Department of Mathematics, Rutgers University, New Brunswick, N.J., USA
}

Received February 2, 1974

\begin{abstract}
A renormalization method originally proposed by Ashmore is reformulated and shown to be, in fact, a renormalization. The method involves use of different complex dimensions associated with various subgraphs of a graph, and appears to combine the best features of complex dimensional and analytic renormalization.
\end{abstract}

In an earlier paper Ashmore [1] proposed an ingenious synthesis of analytic and complex-dimensional renormalization, based on the use of distinct complex dimensions associated with various subgraphs of a Feynman graph. Unfortunately, several errors in this paper necessitate a fairly complete reformulation of the method; one such reformulation is presented here. Our regularization procedure $(\S 1)$ is the same as that of [1], although we give it in an $x$-space version which is needed later. The renormalization procedure, also defined in $\S 1$, differs from that of [1] by taking a different set of parameters as basic. In $\S 2$ we prove that the renormalization has an additive structure, as desired.

\section{Regularization and Renormalization}

Let $G$ be a fixed 2-connected Feynman graph, with vertex set $U_{0}=\left\{V_{1}, \ldots, V_{m}\right\}$ and line set $\mathscr{L}$. We will ignore spin in this paper (although the methods given extend readily) and thus assume that a line $\ell \in \mathscr{L}$ has propagator

$$
\tilde{\Delta}_{\ell}(p)=-i\left(m_{\ell}^{2}-p^{2}-i 0\right)^{-1},
$$

with $m_{\ell}>0$. We first establish our terminology.

If $H$ is a subgraph of $G, N(H)$ denotes the number of loops of $H$, $\mathscr{L}(H)$ the set of lines of $H$. $H$ naturally decomposes into 2-connected subgraphs and single lines joining them; these are called the pieces of $H$. A generalized vertex $U$ is a subset of $U_{0}$, and $G(U)$ denotes the graph which contains all lines joining any pair of vertices in $U$; we will drop the distinction between $U$ and $G(U)$ when no confusion can arise. In

\footnotetext{
* Research sponsored by National Science Foundation, Grant No. NSF-GP-38507.
} 
general, $\mathscr{K}$ will denote a set of 2-connected generalized vertices; in particular $\mathscr{K}[U]=\{W \subset U \mid W$ is 2-connected $\}$, and $\mathscr{K}_{0}=\mathscr{K}\left[U_{0}\right]$. For $\ell \in \mathscr{L}$ with initial and final vertices $V_{i \ell}, V_{f_{\ell}} \in U_{0}, \mathscr{K}_{\ell}=\left\{W \in \mathscr{K}_{0} \mid l \in G(W)\right\}$.

Now, given non-negative integers $\omega_{U}$ for all $U$ in some $\mathscr{K}$, define

$$
M(\mathscr{K})=\prod_{U \in \mathscr{K}} \mathbb{R}^{\omega_{U}} .
$$

$M(\mathscr{K})$ is furnished with Minkowskian inner product in which only the first component in $\mathbb{R}^{\omega_{U_{0}}}$ is timelike [if $\omega_{U_{0}}=0$ or $U_{0} \notin \mathscr{K}$ the inner product is in fact Euclidean, but this does not arise in practice]. For $\mathscr{K}^{\prime} \subset \mathscr{K}$ and $x \in M(\mathscr{K}), x^{\mathscr{K}}$ denotes the $\mathscr{K}^{\prime}$ components of $x$. The Fourier transform on $M(\mathscr{K})$ is

$$
\tilde{f}(p)=(2 \pi)^{-\frac{1}{2} \sum_{\mathscr{K}} \omega_{U}} \int_{M(\mathscr{K})} e^{-i p \cdot x} f(x) d x .
$$

Definition 1.1. For $\ell \in \mathscr{L}$, define a propagator on $M\left(\mathscr{K}_{\ell}\right)$ by

$$
\tilde{\Delta}_{\ell}^{\prime}\left(\lambda_{\ell}\right)(p)=-i\left(m_{\ell}^{2}-p^{2}-i 0\right)^{-\lambda_{\ell}}
$$

$\left(p \in M\left(\mathscr{K}_{\ell}\right)\right)$; define a Feynman amplitude for $G$ on $M\left(\mathscr{K}_{0}\right)^{m}$ by

$$
\mathscr{T}(\lambda, \varrho, \boldsymbol{x})=\prod_{\mathscr{L}} \Delta_{\ell}^{\prime}\left(\lambda_{\ell}\right)\left(x_{f_{\ell}}^{\mathscr{F}_{\ell}}-x_{i_{\ell}}^{\mathscr{K}_{\ell}}\right),
$$

$\boldsymbol{x} \in M\left(\mathscr{K}_{0}\right)^{m}$. Here we have chosen as fundamental variables $\left\{\varrho_{U} \mid U \in \mathscr{K}_{0}\right\}$ defined by

$$
\varrho_{U}=\sum_{\substack{W \supset U \\ W \in \mathscr{K}_{0}}} \omega_{W} .
$$

Remark 1.2. We have introduced the $\lambda$ variables of conventional analytic renormalization both to make (1.1) well defined ( $\Delta_{\ell}^{\prime}$ is continuous for $\operatorname{Re} \lambda_{\ell}$ sufficiently large [2]) and for use in $\S 2$; here we will specialize to $\lambda=\lambda^{0}=(1,1, \ldots, 1)$ before renormalizing. Note that the physical point $\varrho^{0}$ is given by $\varrho_{U}=4, \forall U \in \mathscr{K}_{0}$.

For $U \in \mathscr{K}_{0}$ with $|U|=k$, let $S^{U}=\mathbb{R}^{\frac{1}{2} k(k+1)}$ be the space of variables $\left\{\left(s_{i j}^{U}\right) \mid V_{i}, V_{j} \in U\right\}$, let

and define $\varphi: M\left(\mathscr{K}_{0}\right)^{m} \rightarrow S$ by

$$
S=\prod_{U \in \mathscr{K}_{0}} S^{U},
$$

$$
s_{i j}^{U}=p_{i}^{U} \cdot p_{j}^{U}, \quad\left(V_{i}, V_{j} \in U\right) .
$$

Lemma 1.3. The Fourier transform of (1.1) is

$$
\begin{aligned}
\tilde{\mathscr{T}}(\boldsymbol{\lambda}, \boldsymbol{\varrho}, \boldsymbol{p}) & =\prod_{U \in \mathscr{Y}_{0}}\left\{(2 \pi)^{\frac{1}{2} \omega_{U}} \delta\left(\sum_{V_{i} \in U} p_{i}^{U}\right) \prod_{V_{j} \notin U}(2 \pi)^{\frac{1}{2} \omega_{U}} \delta\left(p_{j}^{U}\right)\right\} \\
& F(\lambda, \varrho, \varphi(\boldsymbol{p}))
\end{aligned}
$$


where

$$
\begin{aligned}
& F(\lambda, \varrho, s) \\
& \quad=f(\lambda, \varrho) \int_{\alpha_{\ell} \geqq 0, \Sigma \alpha_{\ell}=1} \cdots \int_{\mathscr{L}} \prod^{\lambda_{\ell}-1} d \alpha \prod_{\mathscr{K}_{0}} d_{U}(\alpha)^{-\frac{1}{2} \omega_{U}} \\
& \cdot\left[\sum_{\mathscr{L}} m^{2} \alpha_{\ell}-\sum_{\mathscr{K}_{0}} d_{U}^{-1}(\alpha) D_{U}^{s}\left(\boldsymbol{\alpha}, s^{U}\right)-i 0\right]^{-v_{G}} \\
& f(\boldsymbol{\lambda}, \varrho)=(-i)^{m-1} 2^{-\frac{1}{2} \Sigma_{\mathscr{X}_{0}} N(U) \omega_{U}} \Gamma\left(v_{G}\right) \prod_{\mathscr{L}} \Gamma\left(\lambda_{\ell}\right)^{-1}
\end{aligned}
$$

and, for any 2-connected subgraph $H$ of $G$,

$$
v_{H}=\sum_{\mathscr{L}(H)} \lambda_{\ell}-\frac{1}{2} \sum_{\mathscr{K}_{0}} N(U \cap H) \omega_{U} .
$$

Here $d_{U}$ is the usual function for the graph $U$, and $D_{U}^{s}$ is the momentum part of the function $D$ for $U$.

Proof. Use the standard techniques of exponentiation of propagators followed by Gaussian integl ،tion [2].

We note that $F(\lambda, \varrho, s)$ depends on the parameters $\varrho$ only through the explicit analytic dependence on the variables $\omega$ exhibited in (1.4)-(1.6). Thus these equations define $F$ as a function of complex variables $\varrho$. The integral in (1.4) converges only for $\operatorname{Re} \lambda_{\ell}$ sufficiently large (how large depends on $\varrho$ ) but we have

Lemma 1.4. $F(\lambda, \varrho, s)$ is a meromorphic function of $(\lambda, \varrho) \in \mathbb{C}^{|\mathscr{L}|} \times \mathbb{C}^{\left|\mathscr{K}_{0}\right|}$, with simple poles on the varieties

$$
v_{H}=0,-1,-2, \ldots,
$$

for $H$ any 2-connected subgraph of $G$.

Proof. If $\mathscr{E}$ denotes an $s$-family of subgraphs of $G$ ([2]; see also [3], where the analogous concept is that of a labelled $s$-family), we may decompose $F$ as

with

$$
F=\sum_{\mathscr{E}} F_{\mathscr{E}},
$$

$$
\begin{aligned}
F_{\mathscr{E}}= & f(\lambda, \varrho) \int_{0}^{1} \cdots \int_{0}^{1} \prod_{\substack{H \in \mathscr{E} \\
H \neq G}} t_{H}^{v_{H}-1} \prod_{\mathscr{K}_{0}} E_{U}(\boldsymbol{t})^{-\frac{1}{2} \omega_{u}} \\
& \cdot\left[\sum_{\mathscr{L}} m_{\ell}^{2} \alpha_{\ell}(t)-\sum_{\mathscr{K}_{0}} E_{U}^{-1} B_{U}(\boldsymbol{t}, \boldsymbol{s})-i 0\right]^{-v_{G}} .
\end{aligned}
$$

Here $E_{U}$ is non-vanishing for non-negative $t$. Treating $t_{H}^{\nu_{H}-1}$ as a distribution [4], we arrive at the conclusion of the lemma.

Remark 1.5. This is a corrected version of Theorem 2.2 of [1], in which an erroneous formula for $v_{H}$ is given. The error has propagated to an incorrect definition of renormalization, as we discuss below. 
We may now descend from our arbitrarily large space $M\left(\mathscr{K}_{0}\right)$ to a more comfortable environment. Let $M_{n}$ denote $n$-dimensional Minkowski space (first component timelike) and $\varphi_{n}: M_{n}^{m} \rightarrow S$ the map with

For $p \in M_{n}$, let

$$
s_{i j}^{U}=\left\{\begin{array}{lll}
p_{i} \cdot p_{j}, & \text { if } \quad U=U_{0}, \\
0, & \text { if } \quad U \neq U_{0} .
\end{array}\right.
$$

$$
\mathscr{T}_{n}(\lambda, \varrho, p)=(2 \pi)^{n / 2} \delta\left(\sum_{1}^{m} p_{i}\right) F\left(\lambda, \varrho, \varphi_{n}(\boldsymbol{p})\right) .
$$

Then $\mathscr{T}_{4}\left(\lambda^{0}, \varrho, p\right)$ is the regularized amplitude as defined in [1].

For the rest of this section we set $\lambda=\lambda^{0}$. We wish to define a renormalized amplitude by applying an evaluator to $\mathscr{T}_{4}$, as in the case of analytic renormalization $[2,3,5]$. In [1] this is done by taking the variables $\omega$ as fundamental; in fact, however, this does not appear to yield a renormalization. We will modify this approach by working with the variables $\varrho$; our justification is Theorem 2.7 , which states that this procedure yields a renormalization. See also Remark 2.4.

Since (1.2) may be solved for $\omega$ in terms of $\varrho,(1.6)$ becomes

$$
v_{H}=-\frac{1}{2} \sum_{U \in \mathscr{K}_{0}} \beta_{H, U} \varrho_{U}+|\mathscr{L}(H)|
$$

with $\beta_{H, U}$ integer coefficients. This form of singularity is not as simple as that occurring in analytic renormalization (where in effect $\beta_{H, U}$ is always zero or one), but can be treated with a slight modification of the standard evaluator. Suppose that $g(\varrho)$ is a meromorphic function with a finite number of series of singularities of the form

$$
\sum \gamma_{U} \varrho_{U}=k, \quad k \in \mathbb{Z}
$$

with $\gamma_{U}$ an integer. Then for $\left|\varrho_{U}-4\right|<R_{1}$, define

$$
(\mathscr{V} g)(\varrho)=(2 \pi i)^{-K}(K !)^{-1} \sum_{\sigma} \prod_{U \in \mathscr{K}_{0}}\left[\int_{C_{\sigma(U)}} \frac{d \mu_{U}}{\left(\mu_{U}-\varrho_{U}\right)}\right] g(\mu)
$$

where $K=\left|\mathscr{K}_{0}\right|$, the sum is over all $1-1$ onto maps $\sigma: \mathscr{K}_{0} \rightarrow\{1, \ldots, K\}$, and for $1 \leqq i \leqq K, C_{i}$ is the contour $|z-4|=R_{i}$. The $\left\{R_{i}\right\}$ must satisfy

$$
R_{i}>A \sum_{j<i} R_{j}
$$

with $A=\max \left|\gamma_{U}\right|$, the maximum taken over all $U \in \mathscr{K}_{0}$ and all singularities (1.8).

Finally we have

Definition 1.6. The renormalized amplitude for the graph $G$ is

$$
\mathscr{V} \mathscr{T}_{4}\left(\lambda^{0}, \varrho^{0}, p\right)
$$

(where it must be understood that we first set $\lambda=\lambda^{0}$, then apply $\mathscr{V}$, then set $\varrho=\varrho^{0}$ ). 


\section{Structure of the Renormalization}

In this section we show that the renormalization of Definition 1.6 has an additive structure and hence is indeed a renormalization. As was done in [1], we base our proof on the structure of analytic evaluators developed in [5]. In applying this structure, however, we face what appears to be a fundamental complication in complex dimensional regularization - the fact that the regularized amplitude is no longer a product of propagators. Here we treat this difficulty by using the product structure of the regularized amplitude (1.1) in a preliminary stage, then extending results to non-integral $\varrho$ by Carlson's theorem. For an additional discussion of the general character of our result, see Remark 2.8 .

Definition 2.1. For $U \subset U_{0}$ a 2-connected generalized vertex, let $\mathscr{T}_{U}$ denote the amplitude of Definition 1.1 for the graph $G(U)$. Note that $\mathscr{K}_{0}$ will be replaced by $\mathscr{K}[U]$ throughout, so that $\mathscr{T}_{U}$ will depend on $\left\{\varrho_{W} \mid W \in \mathscr{K}[U]\right\}$, and (1.2) will become

$$
\varrho_{W}=\sum_{\substack{X \supset W \\ X \in \mathscr{K}[U]}} \omega_{X} .
$$

$\mathscr{T}_{U}$ will be singular on varieties

$$
v_{H}=\sum_{\mathscr{L}(H)} \lambda_{\ell}-\frac{1}{2} \sum_{W \in \mathscr{K}[U]} N(W \cap H) \omega_{W}=0,-1,-2, \ldots,
$$

for $H$ any 2-connected subset of $G(U)$. The various quantities of $\S 1$ will be subscripted with $U$ when they refer to the graph $G(U)$.

Definition 2.2. For any $\mathscr{K} \in \mathscr{K}[U]$, let

$$
H=\bigcup_{W \in \mathscr{K}} G(W),
$$

and let $P_{\mathscr{K}, U}$ be the partition of $U$ induced by the following equivalence relation: $V_{i} \sim V_{j}$ if $i=j$ or $V_{i}$ and $V_{j}$ lie in the same connected component of $H$. (Note: the union in (2.3) is a true union of graphs, i.e., $\ell \in \mathscr{L}(H)$ iff $\ell \in \mathscr{L}(G(W))$ for some $W \in \mathscr{K}$; thus $H$ is not necessarily a generalized vertex.)

Lemma 2.3. Let $H \subset G$ be 2-connected. Let $U(H)$ be the set of vertices of $H$, and take $U \in \mathscr{K}_{0}$ with $U \supset U(H)$. From (2.1) we have

$$
\sum_{W \in \mathscr{K}[U]} N(H \cap W) \omega_{W}=\sum_{W \in \mathscr{K}[U]} \beta_{H, W} \varrho_{W}
$$

for certain integers $\beta_{H, W}$. Then

(a) $\beta_{H, W}=0$ unless $W \subset U(H)$;

(b) For $W \in U(H), \beta_{H, W}$ is independent of $U$;

(c) If $\mathscr{K}(H)=\left\{W \mid \beta_{H, W} \neq 0\right\}$, then $P_{\mathscr{K}(H), U(H)}=\{U(H)\}$.

Remark 2.4. This lemma demonstrates that the $\varrho$ variables are natural for our problem. For (a) and (b) imply that the form of the singularity 
(2.2) of $\mathscr{T}_{U}$ depends only on $H$, not on $U$; this is not true in terms of the $\omega$ variables. Moreover, the singularity involves only variables associated with $H$ (or $U(H))$; this enables the corresponding singular part for the amplitude $\mathscr{T}$ to be identified as arising from a vertex part of $U(H)$.

Proof of Lemma 2.3. For $W \in \mathscr{K}[U]$, let $W \cap U(H)$ have pieces $H_{i}^{W}$, $i=1, \ldots, k(W)$; for $X \subset U(H)$, define $\tilde{\omega}_{X}=\Sigma \omega_{W}$, the sum running over all $W \in \mathscr{K}[U]$ such that $X \in\left\{H_{i}^{W}\right\}$. Then

and, solving (2.5),

$$
\varrho_{X}=\sum_{X \subset X^{\prime} \in \mathscr{K}[U(H)]} \tilde{\omega}_{X^{\prime}}
$$

$$
\tilde{\omega}_{X}=\sum_{X \subset X^{\prime} \in \mathscr{K}[U(H)]} \alpha_{X, X^{\prime}} \varrho_{X^{\prime}}
$$

where $\alpha_{X, X^{\prime}}$ is independent of $U$. From (2.6),

$$
\begin{gathered}
\sum_{W \in \mathscr{K}[U]} N(H \cap W) \omega_{W}=\sum_{X \in \mathscr{K}[U(H)]} N(H \cap X) \tilde{\omega}_{X} \\
=\sum_{X^{\prime} \in \mathscr{K}[U(H)]}\left[\sum_{X \in \mathscr{K}[U(H)]} N(X \cap H) \alpha_{X, X^{\prime}}\right] \varrho_{X} .
\end{gathered}
$$

$\beta_{H, W}$ is the expression in square brackets in (2.7), and (a) and (b) are immediate.

To prove (c), suppose that $P_{\mathscr{K}(H), U(H)}=\left\{W_{1}, \ldots, W_{r}\right\}$, with $r \geqq 2$. For each $i, 1 \leqq i \leqq r$, set $\omega_{W_{i}}=1, \omega_{W}=0,\left(W \neq W_{i}\right)$ in (2.4) to find $N\left(H \cap W_{i}\right)$ $=\sum_{W \subset W_{i}} \beta_{H, W} ;$ similarly, set $\omega_{U(H)}=1, \omega_{W}=0,(W \neq U(H))$ to find $N(H)=\sum_{W \subset U(H)} \beta_{H, W}$. But then

$$
N(H)=\sum_{i=1}^{r} N\left(H \cap W_{i}\right),
$$

which is impossible for $H$ 2-connected.

Definition 2.5. Following [5], we may use the analytic evaluator $\mathscr{V}$ of (1.9) to define a singular part operator $\mathscr{S}(\mathscr{K})$ for each $\mathscr{K} \subset \mathscr{K}_{0}$, with

$$
\mathscr{V}=\sum_{\mathscr{K} \subset \mathscr{K}_{0}} \mathscr{S}(\mathscr{K})
$$

Then for each $U \subset U_{0}$, and $p \in\left(M_{n}\right)^{|U|}$,

$$
\tilde{\mathscr{Y}}_{U, n}(\varrho, p)=\Sigma \mathscr{S}(\mathscr{K}) \mathscr{T}_{U, n}\left(\lambda^{0}, \varrho, p\right) \text {, }
$$

the sum taken over all $\mathscr{K} \subset \mathscr{K}[U]$ such that $P_{\mathscr{K}, U}=\{U\}$.

Lemma 2.6. $\mathscr{Y}_{U, n}$ is a vertex part for $U$, i.e.,

$$
\tilde{\mathscr{Y}}_{U, n}(\varrho, p)=\delta\left(\sum_{V_{i} \in U} p_{i}\right) D(\boldsymbol{p})
$$

with $D$ an invariant polynomial of degree at most $\mu(G(U))=4 N(G(U))$ $-2|\mathscr{L}(G(U))|$, the superficial divergence in dimension 4. 
Proof. Using the formula

$$
(X-i 0)^{-v}=\lim _{\varepsilon \rightarrow 0+} \Gamma(v)^{-1} \exp \left(\frac{1}{2} v \pi i\right) \int_{0}^{\infty} d t t^{\nu-1} \exp i t(X-i \varepsilon),
$$

we may modify (1.7) to write $F_{U}\left(\lambda^{0}, \varrho, s^{U}\right)$ as a sum [over $s$-families $\mathscr{E}$ of $G(U)]$ of terms

$$
\lim _{\varepsilon \rightarrow 0+} \int_{0}^{\infty} d t_{G(U)} \int_{0}^{1} \cdots \int_{0}^{1} \prod_{\substack{H \in \mathscr{E} \\ H \neq G(U)}} d t_{H} \prod_{H \in \mathscr{E}} t_{H}^{\nu_{H}-1} A_{\varepsilon}\left(\boldsymbol{t}, \boldsymbol{s}^{U}\right) .
$$

If we expand the meromorphic factors $t_{H}^{v_{H}-1}$ around $\varrho^{0}$,

$$
t_{H}^{v_{H}-1}=\frac{\delta^{\left(k_{H}\right)}\left(t_{H}\right)}{\left(v_{H}+k_{H}\right) k_{H} !}+\text { regular part, }
$$

where $2 k_{H}=\mu_{H}$, and apply $\mathscr{S}(\mathscr{K})$, all terms are annihilated except those containing the $\delta$-function factors for a set of graphs $H_{1}, \ldots, H_{p}$ satisfying $\bigcup_{1}^{p} \mathscr{K}\left(H_{i}\right)=\mathscr{K}[$ Remark 3.19 (a) of [5]].

Now note $H_{0}=\bigcup_{1}^{p} H_{i}$ connects all vertices of $U$. For by the condition $P_{\mathscr{K}, U}=\{U\}$, this is true for $H_{0}^{\prime}=\bigcup_{W \in \mathscr{K}} G(W)$, and given $V_{i}, V_{j} \in U$ and a path joining them in $H_{0}^{\prime}$, it may be replaced by a path in $H_{0}$, using Lemma 2.3 (c). If $H_{1}, \ldots, H_{r}$ are the maximal elements among $H_{1}, \ldots, H_{p}$, then also $H_{0}=\bigcup_{i=1}^{r} H_{i}$, moreover $\sum_{1}^{r} k_{H_{i}} \leqq k_{U}$, since the $H_{i}$ are elements of a single $s$-family. It follows as in Lemma 4.2 of [5] that the contribution to (2.9) from any term involving $\prod_{1}^{r} \delta^{\left(k_{H_{i}}\right)}\left(t_{H_{i}}\right)$ is a vertex part for $G(U)$, q.e.d.

For $v \in \mathbb{C}$, let $\varrho(v) \in \mathbb{C}^{\left|\mathscr{K}_{0}\right|}$ be given by $\varrho_{W}=v, W \in \mathscr{K}_{0}$. Then if

$$
\mathscr{F}_{U, n}(\lambda, v, \boldsymbol{p})=\mathscr{T}_{U, n}(\lambda, \varrho(v), \boldsymbol{p}),
$$

$\mathscr{F}_{U, n}\left(\lambda^{0}, v, p\right)$ is the usual complex dimensionally regularized amplitude in dimension $n[6,7]$. Recall also [7] that if $Q=\left\{U_{1}, \ldots, U_{M}\right\}$ is a partition of $U_{0}$, and $\mathscr{X}\left(U_{i}\right)$ is a vertex part for $U_{i}$ [see (2.8)], we can define the regularized amplitude

$$
\mathscr{F}_{Q, X, n}(\lambda, v, \boldsymbol{x}), \quad \boldsymbol{x} \in\left(M_{n}\right)^{m},
$$

given for $v=n$ and $\operatorname{Re} \lambda_{\ell}, \ell \notin \mathscr{L}\left(G\left(U_{i}\right)\right)$, sufficiently large as a product on $\left(M_{n}\right)^{m}$ by

$$
\prod_{\text {conn }} \Delta_{\ell, n}\left(\lambda_{\ell}\right) \prod_{1}^{M} \mathscr{X}\left(U_{i}\right)
$$

and then extended to all $v$ and $\lambda$ as for $\mathscr{T}_{n}$ earlier. 
Theorem 2.7. Let $\mathscr{X}_{U, n}(v, \boldsymbol{p})=\mathscr{Y}_{U, n}(\varrho(v), \boldsymbol{p})$. Then

for all $v \in \mathbb{C}, p \in\left(M_{4}\right)^{m}$.

$$
\left(\mathscr{V}_{4}\right)\left(\lambda^{0}, \varrho(v), \boldsymbol{p}\right)=\sum_{Q} \mathscr{F}_{Q, \mathscr{X}^{\prime}(v), 4}\left(\lambda^{0}, v, \boldsymbol{p}\right)
$$

Remark 2.8. (2.10) expresses the desired subtractive structure of the renormalized amplitude. The unexpected element is that this structure appears to exist only after the specialization $\varrho=\varrho(v)$; we can find no adequate formulation when $\varrho$ is arbitrary. This is in contrast to the situation in analytic renormalization, where the specialization $\lambda=(\lambda, \lambda, \ldots, \lambda)$ is not needed until the implementation of the subtractions via counter terms.

We will prove (2.10) using Carlson's theorem [8,9]; for the estimates involved it will be simplest to work with Euclidean momenta. But note that if we can prove (2.10) with all momenta Euclidean, it will extend to Minkowskian momenta via analytic continuation in the variables $s_{i j}$. For the rest of this section, then, we will assume that $D_{U}^{s}(\boldsymbol{\alpha}, s) \leqq 0$, $B_{U}(t, s) \leqq 0$ [see (1.5), (1.7)].

Lemma 2.9. Let $m_{0}$ be the smallest mass in $G$ and for $U \in \mathscr{K}_{0}$ let

$$
g_{U}(\lambda, \varrho)=\Gamma^{-1}\left(v_{G(U)}\right) \prod_{\mathscr{L}(G(U))} \Gamma\left(\lambda_{\ell}\right)\left(m_{0}^{2}\right)^{v_{G(U)}} .
$$

Then for some constant $K$,

$$
\left|g_{U} F_{U}\right| \leqq K
$$

whenever

$$
\omega_{W} \geqq 0, \quad v_{H} \geqq 1,
$$

for every $W \in \mathscr{K}[U]$ and 2-connected $H \subset G(U)$.

Proof. Under the stated conditions $\left|g_{U} F_{U, \mathscr{E}}\right|<1$ for each $s$-family $\mathscr{E}$ of $G(U)$ [see (1.7)], from which (2.11) follows.

Lemma 2.10. Let $Q=\left\{U_{1}, \ldots, U_{M}\right\}$ be a partition of $U_{0}$, let $\omega_{W}$, $W \in \mathscr{K}_{0}$, satisfy

$$
\omega_{W}=\left\{\begin{array}{l}
n, \quad \text { if } \quad U=U_{0}, \\
0, \quad \text { if } \quad U \neq U_{0}, U \leftarrow \bigcup_{1}^{M} \mathscr{K}\left[U_{i}\right] \\
\text { an arbitrary positive integer, otherwise, }
\end{array}\right.
$$

and let $\varrho_{W}$ be defined by (1.2). Then for $\boldsymbol{x} \in\left(\mathbb{R}^{n}\right)^{m}$,

(a) $\mathscr{T}_{n}(\lambda, \varrho, x)=\prod_{i=1}^{M} \mathscr{T}_{U_{i}, n}(\lambda, \varrho, x) \prod_{\text {conn }} \Delta_{\ell, n}\left(x_{f_{\ell}}-x_{i_{\ell}}\right)$.
(b) (2.14) also holds for $\varrho_{W}, W \subset \bigcup_{1}^{M} \mathscr{K}\left[U_{i}\right]$, arbitrary complex numbers. 
Proof (a). For each $W \in \mathscr{K}\left[U_{i}\right]$, choose a vertex $V_{j(W)} \in W$, multiply (1.1) by

$$
\prod_{i=1}^{M} \prod_{W \in \mathscr{K}\left[U_{i}\right]}(2 \pi)^{-\frac{1}{2} \omega_{W}(|W|-1)} \delta\left(x_{j(W)}^{W}\right) \prod_{j \notin W} \delta\left(x_{j}^{W}\right),
$$

and integrate over all $x_{j}^{W}, j \in U_{0}, W \in \bigcup_{1}^{M} \mathscr{K}\left[U_{i}\right]$. This gives (2.14), as may be seen by going into momentum space. Note that from (2.13), $\Delta_{\ell}^{\prime}=\Delta_{\ell, n}$ for $\ell$ a line in $\Pi_{\text {conn }}$.

(b) Observe that both sides of (2.14) are defined (independently) for complex $\varrho_{W}, W \in \bigcup_{1}^{M} \mathscr{K}\left[U_{i}\right]$; we will use Carlson's theorem to prove that the equality which holds for certain integer values of $\varrho_{W}$ extends to complex values. Multiply both sides of $(2.14)$ by $g_{U_{0}}(\lambda, \varrho)$, take the $\mathbb{R}^{n}$ Fourier transform, and drop the resulting factor $\delta\left(\Sigma_{U_{0}} p_{i}\right)$. In the region (2.12), the left hand side is bounded by a constant (Lemma 2.9), and using

$$
\left|g_{U_{\imath}} F_{U_{i}}\right| \leqq K_{i}
$$

(Lemma 2.9), the convolutions on the right hand side may be worked out to show that the right hand side is bounded by

$$
g_{U_{0}}(\lambda, \varrho) \prod_{i=1}^{M} K_{i} g_{U_{i}}(\lambda, \varrho)^{-1} F_{\tilde{G}}(\lambda, \varrho(n), s),
$$

where $\tilde{G}$ is the graph obtained from $G$ by contracting all $G\left(U_{i}\right)$.

We now claim that (2.14) holds for all complex $\omega_{W}$ (equivalently, all complex $\left.\varrho_{W}\right), W \in \bigcup \mathscr{K}\left[U_{i}\right]$. For choosing $W \in \mathscr{K}\left[u_{i}\right]$, we fix $n$, $\omega_{U}(U \neq W)$, and certain numbers $\eta_{\ell} \gg 0, l \in \mathscr{L}$. Now set $\lambda_{\ell}=\eta_{\ell} \omega_{W}$; for all $\operatorname{Re} \omega_{W}>\varepsilon>0,(2.12)$ will be satisfied, and (2.15) will be polynomially bounded in $\omega_{W}$ (use Stirling's formula). Hence by Carlson's Theorem, equality holds for all values of $\omega_{W}$, and since the $\eta_{\ell}$ can vary over a real neighborhood, equality holds for all $\lambda_{\ell}$. Repeating the argument for all $W \in \mathscr{K}\left[U_{i}\right]$ proves the Lemma.

Lemma 2.11. Let $Q=\left\{U_{1}, \ldots, U_{m}\right\}$ as above, and let $\lambda_{\ell}=1$, $\ell \in \mathscr{L}\left(G\left(U_{i}\right)\right)$. For $v \in \mathbb{C}$, let $\varrho_{U}=v$ if $U \notin \bigcup_{1} \mathscr{K}\left[U_{i}\right]$.

(a) If $v=n$, a positive integer,

$$
\sum_{\left\{\mathscr{C} \mid P \mathscr{X}, U_{0}=Q\right\}} \mathscr{S}(\mathscr{K}) \mathscr{T}_{n}(\lambda, \varrho, \boldsymbol{x})=\mathscr{F}_{Q, \mathscr{Y}(\varrho), n}(\lambda, n, \boldsymbol{x}) .
$$

(b) For any $v \in \mathbb{C}$,

$$
\sum_{\left\{\mathscr{K} \mid P_{\mathscr{X},}, U_{0}=Q\right\}} \mathscr{S}(\mathscr{K}) \mathscr{T}_{4}(\lambda, \varrho, \boldsymbol{x})=\mathscr{F}_{Q, \mathscr{y}(\boldsymbol{\varrho}), 4}(\lambda, v, \boldsymbol{x}) .
$$


Proof. (a) If $P_{\mathscr{K}, U_{0}}=Q, \mathscr{K}$ must decompose as $\mathscr{K}=\bigcup_{1}^{M} \mathscr{K}_{i}$; with $\mathscr{K}_{i} \subset \mathscr{K}\left[U_{i}\right], P_{\mathscr{K}_{i}, U_{i}}=\left\{U_{i}\right\}$. We set $\lambda_{\ell}=1\left(\ell \in \bigcup \mathscr{L}\left(G\left(U_{i}\right)\right)\right)$ in $(2.14)$ and apply $\mathscr{S}(\mathscr{K})$; this makes sense because $\mathscr{S}(\mathscr{K})$ operates only on variables $\varrho_{W}, W \in \bigcup \mathscr{K}\left[U_{i}\right]$, and these may be taken complex by Lemma 2.10 (b). By the factorization property of $\mathscr{S}(\mathscr{K})$ (Remark 3.19 (b) of [5]), this gives

$$
\mathscr{S}(\mathscr{K}) \mathscr{T}_{n}(\lambda, \varrho, x)=\prod_{i=1}^{M} \mathscr{S}\left(\mathscr{K}_{i}\right) \mathscr{T}_{U_{\imath}, n}\left(\lambda^{0}, \varrho, x\right) \prod_{\text {conn }} \Delta_{\ell, n}\left(\lambda_{\ell}\right) .
$$

Summing over $\mathscr{K}$ satisfying $P_{\mathscr{K}, U_{0}}=Q$ gives (2.16).

(b) This is proved by another application of Carlson's theorem. We omit details, as the argument is similar to the proof of Lemma 2.10 (b).

Proof of Theorem 2.7. Set $\lambda=\lambda^{0}$ and $\varrho=\varrho(v)$ in (2.17), and sum over all partitions $Q$.

\section{References}

1. Ashmore, J.F.: Commun. math. Phys. 29, 177-187 (1973)

2. Speer,E.: Lectures on analytic renormalization. University of Maryland Technical Report 73-067

3. Speer,E.: Generalized Feynman amplitudes. Princeton: Princeton University Press 1969

4. Gelfand,I.M., Shilov, G.E.: Generalized functions, Vol. I. New York: Academic Press 1964

5. Speer, E.: Commun. math. Phys. 23, 23-36 (1971); 25, 336 (1972)

6. t'Hooft, G., Veltman, M.: Nucl. Phys. B-44, 189 (1972)

7. Speer,E.: Renormalization and Ward identities using complex space-time dimension. J. Math. Phys. 35, 151-154 (1974)

8. Squires, E.J.: Complex angular momenta and particle physics. New York: Benjamin 1963

9. Titchmarsh,E.C.: Theory of functions. New York: Oxford University Press 1969

Communicated by K. Symanzik

Eugene R. Speer

Department of Mathematics

Rutgers University

New Brunswick, N.J. 08903, USA 